\title{
The Pentagram Integrals on Inscribed Polygons
}

\author{
Richard Evan Schwartz * Serge Tabachnikov \\ Submitted: Oct 19, 2010; Accepted: Aug 20, 2011; Published: Sep 2, 2011 \\ Mathematics Subject Classification: $37 \mathrm{~J} 35$
}

\begin{abstract}
The pentagram map is a completely integrable system defined on the moduli space of polygons. The integrals for the system are certain weighted homogeneous polynomials, which come in pairs: $E_{1}, O_{2}, E_{2}, O_{2}, \ldots$ In this paper we prove that $E_{k}=O_{k}$ for all $k$, when these integrals are restricted to the space of polygons which are inscribed in a conic section. Our proof is essentially a combinatorial analysis of the integrals.
\end{abstract}

\section{Introduction}

The pentagram map is a geometric iteration defined on polygons. This map is defined over any field, but it is most easily described for polygons in the real projective plane. Geometrically, the pentagram map carries the polygon $P$ to the polygon $Q$, as shown in Figure 1.

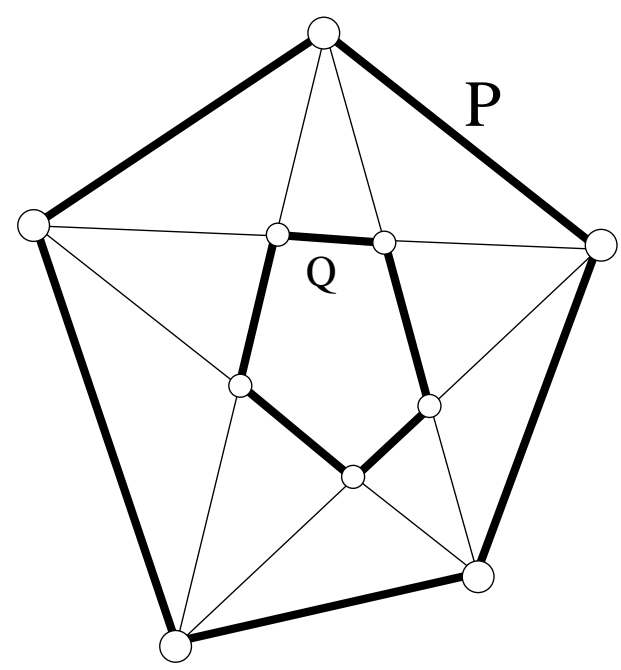

Figure 1: The pentagram map

\footnotetext{
* Supported by N.S.F. Research Grant DMS-0072607
} 
The first reference we know, to some version of the pentagram map, is $[\mathbf{M}]$, where it is studied for pentagons. The first author of this paper wrote a series of papers $[\mathbf{S 1}],[\mathbf{S 2}]$, and [S3] about the map, as defined for general $n$-gons. See also the recent papers $[\mathbf{B}]$, [G], [OST1], [OST2], [Sol] and [ST].

The pentagram map is always defined for convex polygons, and generically defined for all polygons. The pentagram map commutes with projective transformations and induces a generically defined map $T$ on the space $\mathcal{Q}_{n}$ of cyclically labelled 1 projective transformations. $T$ is periodic for $n=5,6$ but not periodic for $n \geq 7$.

In $[\mathbf{S 3}]$ we introduced a larger space $\mathcal{P}_{n}$ of so-called twisted $n$-gons, and then produced polynomials

$$
O_{1}, \ldots, O_{[n / 2]}, O_{n}, E_{1}, \ldots, E_{[n / 2]}, E_{n}: \mathcal{P}_{n} \rightarrow \boldsymbol{R}
$$

which are invariant under the pentagram map. We call these polynomials the monodromy invariants. These invariants restrict to give invariants on $\mathcal{Q}_{n}$. See \$2 for all relevant definitions. The purpose of this paper is to prove the following result, which we noticed numerically a long while ago.

Theorem $1.1 O_{k}(P)=E_{k}(P)$ for any $n$-gon $P$ that is inscribed in a conic section and any $k=1, \ldots,[n / 2], n$.

This result holds equally well for twisted polygons.

We view Theorem 1.1 as part of the ongoing effort to understand the structure of the pentagram map. Here we put Theorem 1.1 in context. In [OST1] we constructed an invariant Poisson bracket on $\mathcal{P}_{n}$, which is compatible with the monodromy invariants. Using these two compatible structures, we showed in [OST1] that the pentagram map is a completely integrable system on $\mathcal{P}_{n}$. We worked with $\mathcal{P}_{n}$ rather than with $\mathcal{Q}_{n}$ because the integrability question is easier there. In our recent paper [OST2], we show that the pentagram map is, in fact, completely integrable on $\mathcal{Q}_{n}$, the main space of interest. The equally recent paper [Sol] establishes this same result independently, by other methods. In practical terms, this means that (at least for convex polygons) the generic orbit is dense in a finite union of tori, and these tori have canonical flat structures which are invariant under the pentagram map.

The pentagram map has connections to other subjects.

- Integrable PDEs: As is explained in [S3], and in more detail in [OST1], the pentagram map is a discretization of the Boussoinesq equation, a well-known integrable partial differential equation. The Poisson bracket in [OST1] is a discretization of an invariant bracket that arises in connection with the Boussoinesq equation, and the monodromy invariants are $2^{2}$ discrete versions of the integrable hierarchy for the Boissoinesq equation. See $[\mathbf{B}]$ for recent generalizations in this direction.

\footnotetext{
${ }^{1}$ Technically, one needs to consider the square of the map in order to get a canonically defined map on labelled $n$-gons. However, if one is willing to break symmety, preferring (say) left over right, then the map itself is defined on labelled $n$-gons.

${ }^{2}$ We have not yet worked out the precise connection between the monodromy invariants and the Boussoinesq hierarchy.
} 
- Cluster Algebras: In [S3] we explained the connection between the pentagram map and the so-called octahedral recurrence. This connection is explored more deeply, and from a different point of view, in $[\mathbf{G}]$, where the pentagram map is shown to be an example of a cluster algebra.

- Configuration Theorems: The pentagram map seems to interact nicely with polygons that are inscribed in conic sections. We mention our paper $[\mathbf{S T}]$, in which we describe some finite configuration theorems, à la Pappus, that we discovered in connection with the pentagram map and inscribed polygons.

- Algebraic integrability: A recent paper [Sol] describes the pentagram map as a discrete zero-curvature equation with a spectral parameter and the dynamics as a linear dynamics on the Jacobian of the spectral curve.

Given the connections between the pentagram map and both integrable systems and cluster algebras, as well as the beauty of the map as a stand-alone object, it seems worthwhile getting information about the nature of the monodromy invariants.

Theorem 1.1 boils down to a countably infinite family of polynomial identities. The polynomials involved are somewhat reminiscent of the symmetric polynomials, but they have somewhat less symmetry then these. One novel feature of the theorem is that we discovered not just the result but also the proof by way of computer experimentation. We wrote a Java program to aid us with the combinatorics of the proof. This applet is available on the first author's website. Download the program at

http://www . math. brown.edu/ res/Java/OEAPPLET.tar

The program has a README file with basic instructions.

While our proof is mainly combinatorial, we think that perhaps there should be a better proof based on geometry. Accordingly, we will describe our polynomials in three ways - geometrically, combinatorially, and in terms of determinants. We will only use the combinatorial description in our proof, but we hope that the other descriptions might ring a bell for some readers. We tried quite hard to find a simple proof of Theorem 1.1] but nothing seemed to work. We invite the reader to look for a simple proof!

Here is an overview of the paper. In $\S 2$ we define twisted polygons and the monodromy invariants. In $\S 3$ we reduce Theorem 1.1 to a combinatorial problem. In $\S 4$ we solve this combinatorial problem.

The second author would like to thank Brown University for its hospitality during his sabbatical.

\section{The Monodromy Invariants}

The papers [S3] and [OST1] give a good account of the monodromy invariants. We will follow $3^{3}$ the notation of [OST1] for this paper. We will give three descriptions of

\footnotetext{
${ }^{3}$ The notation in the two papers is slightly different. After some detailed consideration of this matter, we have decided that in future papers we will revert to the notation in $[\mathbf{S 3}]$ because it is more symmetric. However, we made that decision after adapting everything in this paper to the notation in [OST1].
} 
these invariants, one geometrical, one combinatorial, and one based on determinants. The reader only interested in the proof of Theorem 1.1 need only pay attention to the combinatorial definition. As we say in the introduction, we mention the other definitions just in the hopes that it will ring a bell for some readers.

Because we are not using the geometric or determinental definitions in our proof, we will not include the arguments that show the equivalence of the various definitions. The paper [S3] has a proof that the geometric and combinatorial definitions coincide.

\subsection{The Geometric Definition}

Let $\boldsymbol{R} \boldsymbol{P}^{2}$ denote the real projective plane. All of what we say works over any field, but we find it convenient to restrict our attention to $\boldsymbol{R}$.

A twisted $n$-gon is a map $\phi: \boldsymbol{Z} \rightarrow \boldsymbol{R} \boldsymbol{P}^{2}$ such that

$$
\phi(k+n)=M \circ \phi(k) \quad \forall k .
$$

for some projective transformation $T$. Here $M$ does not depend on $k$. When $M$ is the identity, the notion of a twisted $n$-gon translates in an obvious way into the notion of an ordinary polygon. The map $T$ is called the monodromy of $\phi$.

Two twisted $n$-gons $\phi_{1}$ and $\phi_{2}$ are equivalent if there is some projective transformation $S$ such that $S\left(\phi_{1}\right)=\phi_{2}$. In this case, we have the equation $S M_{1} S^{-1}=M_{2}$. In other words, the monodromies of two equivalent twisted polygons are conjugate. We let $\mathcal{P}_{n}$ denote the space of twisted $n$-gons.

We have the inverse cross ratio

$$
\left[t_{1}, t_{2}, t_{3}, t_{4}\right]=\frac{\left(t_{1}-t_{2}\right)\left(t_{3}-t_{4}\right)}{\left(t_{1}-t_{3}\right)\left(t_{2}-t_{4}\right)}
$$

Suppose that $\phi$ is a twisted $n$-gon, with monodromy $M$. We let $v_{i}=\phi(i)$. The label $i$ in Figure 2 denotes $v_{i}$, and similarly for the other labels.

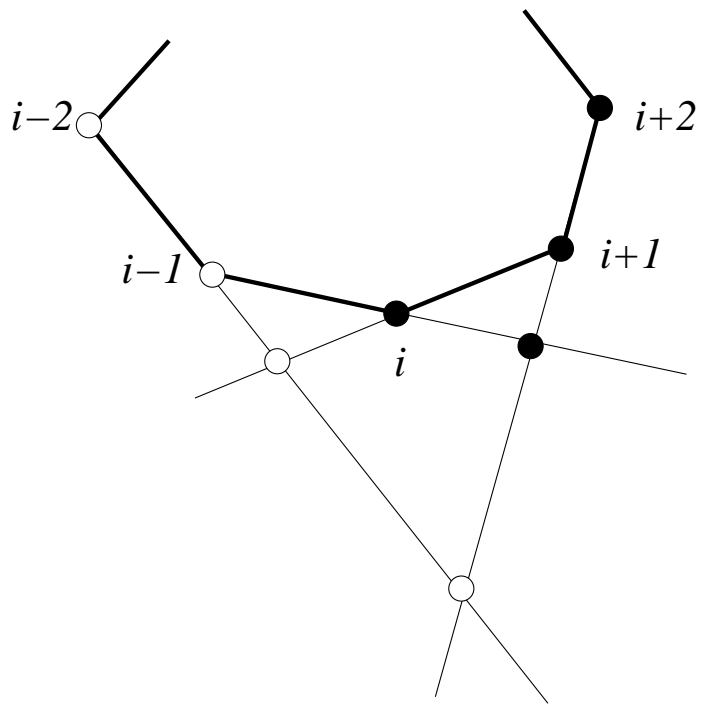

Figure 2: vertex labels 
We associate to each vertex $v_{i}$ two numbers:

$$
\begin{aligned}
& x_{i}=\left[v_{i-2}, v_{i-1},\left(\left(v_{i-2}, v_{i-1}\right) \cap\left(v_{i}, v_{i+1}\right),\left(\left(v_{i-2}, v_{i-1}\right) \cap\left(v_{i+1}, v_{i+2}\right)\right],\right.\right. \\
& y_{i}=\left[v_{i+2}, v_{i+1},\left(\left(v_{i+2}, v_{i+1}\right) \cap\left(v_{i}, v_{i-1}\right),\left(\left(v_{i+2}, v_{i+1}\right) \cap\left(v_{i-1}, v_{i-2}\right)\right] .\right.\right.
\end{aligned}
$$

Here $(a, b)$ denotes the line determined by points $a$ and $b$. For instance, $x_{i}$ is the inverse cross ratio of the 4 white points in Figure 2. We call the invariants $4 x_{1}, y_{1}, x_{2}, y_{2}, \ldots$ the corner invariants. These invariants form a periodic sequence of length $2 n$. That is $x_{k+n}=x_{k}$ and $y_{k+n}=y_{k}$ for all $k$.

We define

$$
O_{n}=\prod_{i=1}^{n} x_{i} ; \quad E_{n}=\prod_{i=1}^{n} y_{i} .
$$

The other monodromy invariants are best defined in an indirect way. Recall that $M$ is the monodromy of our twisted polygon $\phi$. We lift $M$ to an element of $G L_{3}(\boldsymbol{R})$ which we also denote by $M$. We define

$$
\Omega_{1}=\frac{\operatorname{trace}^{3}(M)}{\operatorname{det}(M)} ; \quad \Omega_{2}=\frac{\operatorname{trace}^{3}\left(M^{-1}\right)}{\operatorname{det}\left(M^{-1}\right)} .
$$

These quantities are independent of the lift of $M$ and only depend on the conjugacy class of $M$. Finally, these quantities are invariant under the pentagram map.

We define

$$
\widetilde{\Omega}_{1}=O_{n}^{2} E_{n} \Omega_{1} ; \quad \widetilde{\Omega}_{2}=O_{n} E_{n}^{2} \Omega_{2} .
$$

It turns out that these quantities are polynomials in the corner invariants. The remaining monodromy invariants are suitably weighted homogeneous parts of these polynomials.

We have a basic rescaling operation

$$
R_{t}\left(x_{1}, y_{1}, x_{2}, y_{2}, \ldots\right)=\left(t x_{1}, t^{-1} y_{1}, t x_{2}, t^{-1} y_{2}, \ldots\right)
$$

We say that a polynomial in the corner invariants has weight $k$ if

$$
R_{t}^{*}(P)=t^{k} P
$$

Here $R_{t}^{*}$ denotes the obvious action of $R_{t}$ on polynomials. In [S3] we show that

$$
\widetilde{\Omega}_{1}=\left(\sum_{k=0}^{[n / 2]} O_{k}\right)^{3} ; \quad \widetilde{\Omega}_{2}=\left(\sum_{k=0}^{[n / 2]} E_{k}\right)^{3} .
$$

Here $O_{k}$ and $E_{k}$ are the weight $k$ polynomials in each sum, and $[n / 2]$ denotes the floor of $n / 2$. Note that $O_{0}$ and $E_{0}$ are just the constant function 1.

\footnotetext{
${ }^{4}$ The notation in $[\mathbf{S 3}]$ uses $x_{1}, x_{2}, x_{3}, x_{4}, \ldots$ in place of $x_{1}, y_{1}, x_{2}, y_{2} \ldots$ As we remarked in a previous footnote, our future papers will revert to the notation in $[\mathbf{S 3}]$.
} 


\subsection{The Combinatorial Definition}

In everything we say, the indices are taken cyclically, $\bmod n$. We introduce the monomials

$$
X_{i}:=x_{i} y_{i} x_{i+1} \text {. }
$$

The monodromy polynomial $O_{k}$ is built from the monomials $x_{i}$ for $i=1, \ldots, n$ and $X_{j}$ for $j=1, \ldots, n$. We call two monomials consecutive if they involve consecutive or coinciding variables $x_{i}$. More explicitly, we have the following criteria:

1. $X_{i}$ and $X_{j}$ are consecutive if $j \in\{i-2, i-1, i, i+1, i+2\}$;

2. $X_{i}$ and $x_{j}$ are consecutive if $j \in\{i-1, i, i+1, i+2\}$;

3. $x_{i}$ and $x_{j}$ are consecutive if $j \in\{i-1, i, i+1\}$.

Let $O$ be a monomial obtained by the product of the monomials $X_{i}$ and $x_{j}$, that is,

$$
O=X_{i_{1}} \cdots X_{i_{s}} x_{j_{1}} \cdots x_{j_{t}}
$$

Such a monomial is called admissible if no two of the monomials are consecutive. For every admissible monomial, define the weight $|O|=s+t$ and the $\operatorname{sign} \operatorname{sign}(O)=(-1)^{t}$. The sign just depends on the number of $x_{i}$ singletons appear in the monomial. We have

$$
O_{k}=\sum_{|O|=k} \operatorname{sign}(O) O ; \quad k \in\left\{1,2, \ldots,\left[\frac{n}{2}\right]\right\} .
$$

For example, if $n \geq 5$ we obtain the following two polynomials:

$$
O_{1}=-\sum_{i} x_{i}+\sum_{i} x_{i} y_{i} x_{i+1}, \quad O_{2}=\sum_{|i-j| \geq 2} x_{i} x_{j}-\sum_{|j-i-0.5| \geq 2.5}\left(x_{i} y_{i} x_{i+1}\right) x_{j+1}
$$

The same formulas work for $E_{k}$, if we make all the same definitions with $x$ and $y$ interchanged. More precisely, one builds the polynomials $E_{k}$ from the monomials $y_{i}$ and $Y_{j}:=y_{j-1} x_{j} y_{j}$ with the same restriction that no consecutive monomials are allowed and the same definitions of the weight and sign.

We note that the dihedral symmetry

$$
\sigma\left(x_{i}\right)=y_{-i}, \sigma\left(y_{i}\right)=x_{-i}
$$

interchanges the polynomials $O_{k}$ and $E_{k}$.

A Sign Change: In this paper we will work with variants of the polynomials above. The polynomials

$$
O_{k}^{*}=(-1)^{k} O_{k}, \quad E_{k}^{*}=(-1)^{k} E_{k}
$$

have certain advantages over $O_{k}$ and $E_{k}$ though, of course, they carry the same information. It seems (but we do not know a proof) that $O_{k}^{*}$ and $E_{k}^{*}$ are always positive on convex polygons. On the other hand, these alternate definitions do not interact as gracefully as certain constructions in [S3] and in our forthcoming paper [OST2]. In this paper, we will work with the invariants $O_{k}^{*}$ and $E_{k}^{*}$, for no other reason that the fact that we set up all our notation to work with these versions. 


\subsection{The Determinantal Definition}

Now we describe determinantal formulas for the monodromy invariants; these formulas did not appear in our previous papers on the subject.

For positive integers $k>l$, we define the four-diagonal determinant

$$
F_{l}^{k}=\left|\begin{array}{ccccccc}
1 & x_{k} & X_{k-1} & 0 & 0 & \ldots & 0 \\
1 & 1 & x_{k-1} & X_{k-2} & 0 & \ldots & 0 \\
0 & 1 & 1 & x_{k-2} & X_{k-2} & \ldots & 0 \\
\ldots & \ldots & \ldots & \ldots & \ldots & \ldots & \ldots \\
0 & \ldots & \ldots & \ldots & 1 & 1 & x_{l+1} \\
0 & \ldots & \ldots & \ldots & 0 & 1 & 1
\end{array}\right|,
$$

where $x_{i}$ and $y_{i}$ are the corner invariants and $X_{i}$ is as in (2). By convention,

$$
F_{k+2}^{k}=0, F_{k+1}^{k}=1, F_{k}^{k}=1 \text {. }
$$

Then one has the following formula for the monodromy invariants $O_{k}$ :

$$
\sum_{i=0}^{[n / 2]} O_{i}=F_{1}^{n}+F_{0}^{n-1}-F_{1}^{n-1}+x_{n} y_{n} x_{1} F_{2}^{n-1} .
$$

Similarly, for $E_{k}$, define

$$
G_{p}^{q}=\left|\begin{array}{ccccccc}
1 & y_{p+1} & Y_{p+2} & 0 & 0 & \ldots & 0 \\
1 & 1 & y_{p+2} & Y_{p+3} & 0 & \ldots & 0 \\
0 & 1 & 1 & y_{p+3} & Y_{p+4} & \ldots & 0 \\
\ldots & \ldots & \ldots & \ldots & \ldots & \ldots & \ldots \\
0 & \ldots & \ldots & \ldots & 1 & 1 & y_{q} \\
0 & \ldots & \ldots & \ldots & 0 & 1 & 1
\end{array}\right| .
$$

Then one has:

$$
\sum_{i=0}^{[n / 2]} E_{i}=G_{0}^{n-1}+G_{1}^{n}-G_{1}^{n-1}+y_{n} x_{1} y_{1} G_{1}^{n-2} .
$$

Formulas (5) and (6) simplify if one considers an open version of the monodromy invariants: instead of having a periodic "boundary condition" $x_{i+n}=x_{i}, y_{i+n}=y_{i}$, assume that $x_{i}=0$ for $i \leq 0$ and $i \geq n+1$, and $y_{i}=0$ for $i \leq-1$ and $i \geq n$. With this "vanishing at infinity" boundary conditions, the monodromy invariants are given by a single determinant:

$$
\sum_{i=0}^{[n / 2]} O_{i}=F_{0}^{n}, \quad \sum_{i=0}^{[n / 2]} E_{i}=G_{-1}^{n-1} .
$$




\section{Reduction to the Puzzle}

Let $\boldsymbol{R} \boldsymbol{P}^{2}$ be the real projective plane and let $\boldsymbol{R} \boldsymbol{P}^{1}$ denote the projective line. Everything we say works either in the context of ordinary polygons or twisted polygons. For brevity, we will just say polygon.

A non-degenerate conic in $\boldsymbol{R} \boldsymbol{P}^{2}$ can be identified with $\boldsymbol{R} \boldsymbol{P}^{1}$ by way of the stereographic projection from a point of the conic. This identification is unique up to a projective transformation of the real projective line. That is, a different choice of the center of projection amounts to a projective transformation of $\boldsymbol{R} \boldsymbol{P}^{1}$. If $\left(\ldots, v_{-1}, v_{0}, v_{1}, \ldots\right)$ is an inscribed polygon, we can consider the vertices $v_{i}$ as points of the real projective line and talk about their cross-ratios which are uniquely defined. Referring to the cross-ratio on the projective line, we set:

$$
p_{i}=1-\left[v_{i-2}, v_{i-1}, v_{i}, v_{i+1}\right] .
$$

In the next lemma we express the corner invariants of an inscribed polygon in terms of the quantities $p_{i}$. Once we specify the inverse cross ratios $\left\{p_{i}\right\}$, we produce an inscribed twisted polygon having corner invariants $\left\{\left(x_{i}, y_{i}\right)\right\}$. Thus we have a map $\left(p_{i}\right) \mapsto\left(x_{i}, y_{i}\right)$. We denote this map by $F$.

Lemma 3.1 One has:

$$
x_{i}=\left[v_{i-2}, v_{i-1}, v_{i}, v_{i+2}\right], \quad y_{i}=\left[v_{i-2}, v_{i}, v_{i+1}, v_{i+2}\right]
$$

and the map $F$ is given by the formula

$$
x_{i}=\frac{1-p_{i}}{p_{i+1}}, \quad y_{i}=\frac{1-p_{i+1}}{p_{i}} .
$$

Proof: Consider Figure 3. Using the projection from point $v_{i+1}$, we obtain:

$$
\begin{aligned}
x_{i}=\left[v_{i-2}, v_{i-1}, A, B\right]= & {\left[\left(v_{i+1} v_{i-2}\right),\left(v_{i+1} v_{i-1}\right),\left(v_{i+1} A\right),\left(v_{i+1} B\right)\right]=} \\
& {\left[v_{i-2}, v_{i-1}, v_{i}, v_{i+2}\right] . }
\end{aligned}
$$

A similar projection from $v_{i-1}$ yields the formula for $y_{i}$. The expression for $x_{i}$ in terms of $p_{i}$ and $p_{i+1}$ follows now from the identity

$$
\left[v_{i-2}, v_{i-1}, v_{i}, v_{i+2}\right]=\frac{\left[v_{i-2}, v_{i-1}, v_{i}, v_{i+1}\right]}{1-\left[v_{i-1}, v_{i}, v_{i+1}, v_{i+2}\right]}
$$

and likewise for $y_{i}$. 


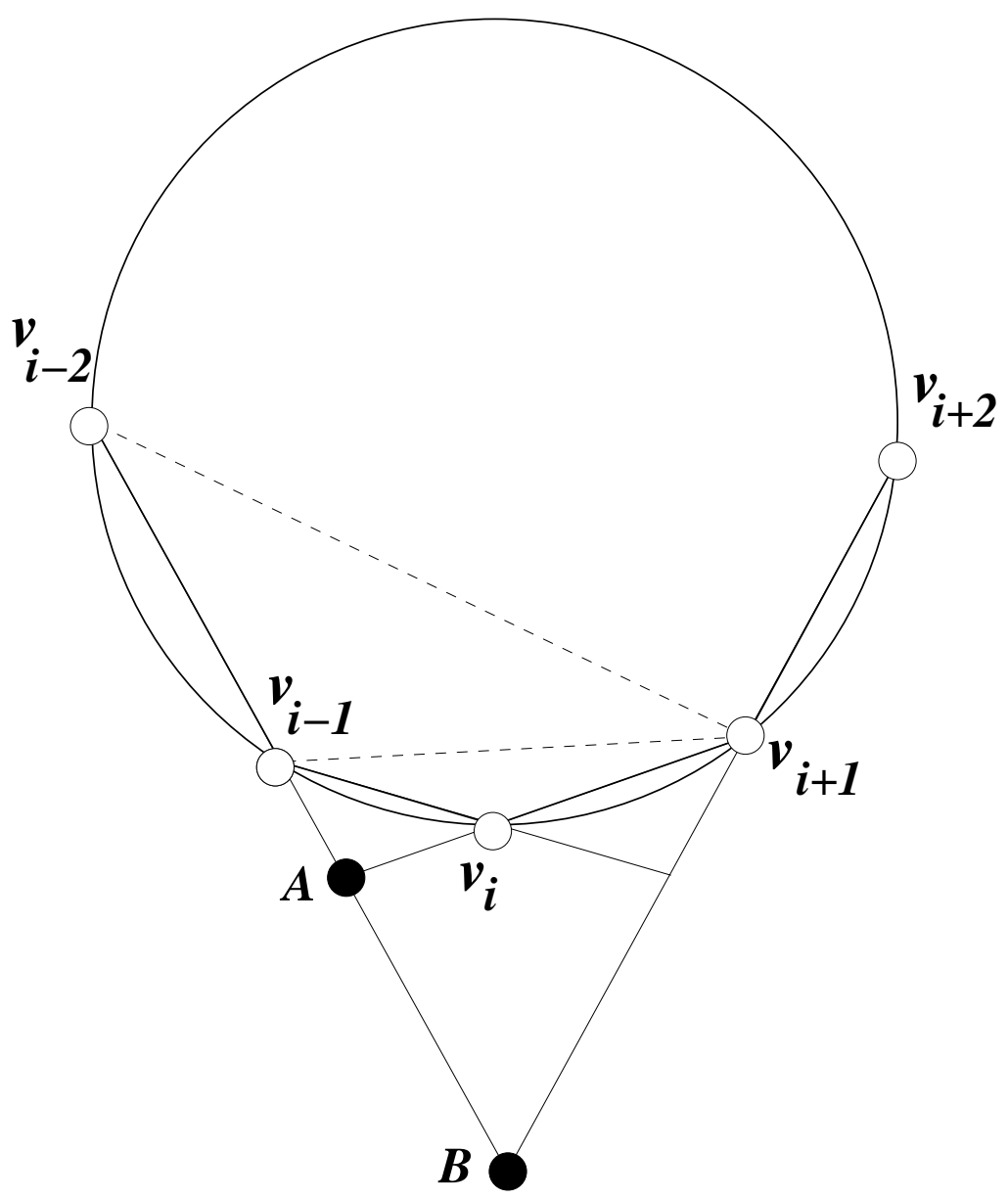

Figure 3: Proof of Lemma 3.1

The dihedral group also acts on cyclic sequences $\left(p_{i}\right)$ by cyclic permutations and the orientation-reversing involution $\sigma^{\prime}\left(p_{i}\right)=p_{1-i}$. It follows from (77) that $F \circ \sigma^{\prime}=\sigma \circ F$ where $\sigma$ is the involution (3). Hence $F$ is a dihedrally equivariant map.

As we remarked in the previous chapter, we will work with $O_{k}^{*}$ and $E_{k}^{*}$ in place of $O_{k}$ and $E_{k}$. After $x_{i}$ and $y_{i}$ are replaced by $p_{i}$ via (7), the polynomials $O_{k}^{*}$ and $E_{k}^{*}$ become Laurent polynomials in the variables $p_{i}$. The identity $E_{n}^{*}=O_{n}^{*}$ obviously holds since both sides equal $\Pi\left(1-p_{i}\right) / p_{i}$. We need to prove that $E_{k}^{*}=O_{k}^{*}$ for $k=1, \ldots,[n / 2]$. We will prove the following result.

Theorem 3.2 (Dihedral Balance). If two monomials in the variables $p_{i}$ are related by an orientation-reversing dihedral symmetry (for example, the involution $\sigma^{\prime}$ ) then they appear in $O_{k}^{*}$ with the same coefficients.

Proof of Theorem 1.1; Since the map $F$ is dihedrally equivariant and the orientationreversing involution on the variables $\left(x_{i}, y_{i}\right)$ interchanges $E_{k}^{*}$ and $O_{k}^{*}$, Theorem 3.2 shows that $O_{k}^{*}(P)=E_{k}^{*}(P)$ for any inscribed polygon $P$. But then $O_{k}(P)=E_{k}(P)$ as well. 
The remainder of the paper is devoted to proving Theorem 3.2. Let us compute the monomials of $O_{k}^{*}$ in terms of the $p$-variables. We find that

$$
x_{i}=\frac{1}{p_{i+1}}-\frac{p_{i}}{p_{i+1}}
$$

and

$$
-X_{i}=-\frac{1}{p_{i} p_{i+1} p_{i+2}}+\frac{1}{p_{i+1} p_{i+2}}+2 \frac{1}{p_{i} p_{i+2}}-2 \frac{1}{p_{i+2}}-\frac{p_{i+1}}{p_{i} p_{i+2}}+\frac{p_{i+1}}{p_{i+2}} .
$$

We see that the variables $p$ 's that appear in $x_{i}$ involve two indices, $i$ and $i+1$, and the those in $X_{i}$ involve three indices, $i, i+1$ and $i+2$. Pictorially, these terms can be represented as follows: for $x_{i}$, see Figure 4 , and for $X_{i}$, see Figure 5 . In these figures, the presence of each term $p$ in the numerator and denominator is represented by a shaded square, and its absence by a white square.
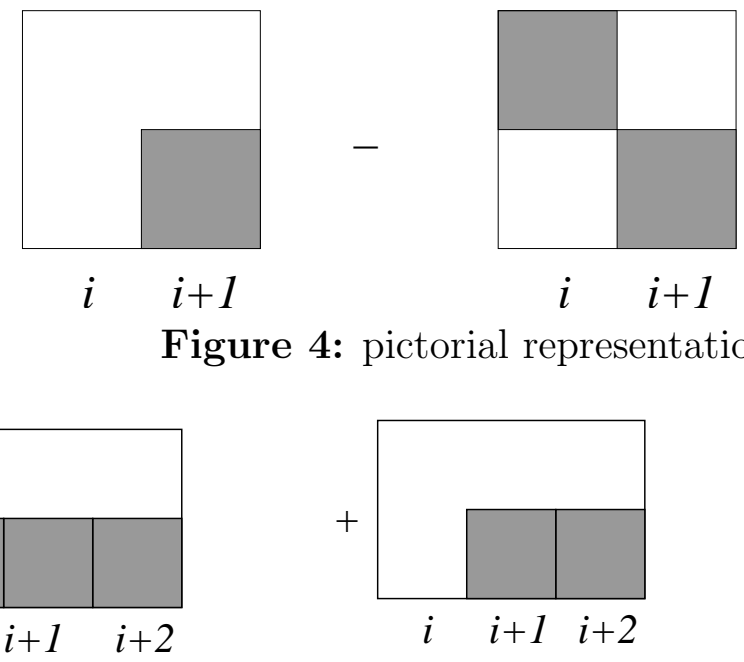

Figure 4: pictorial representation of (8)
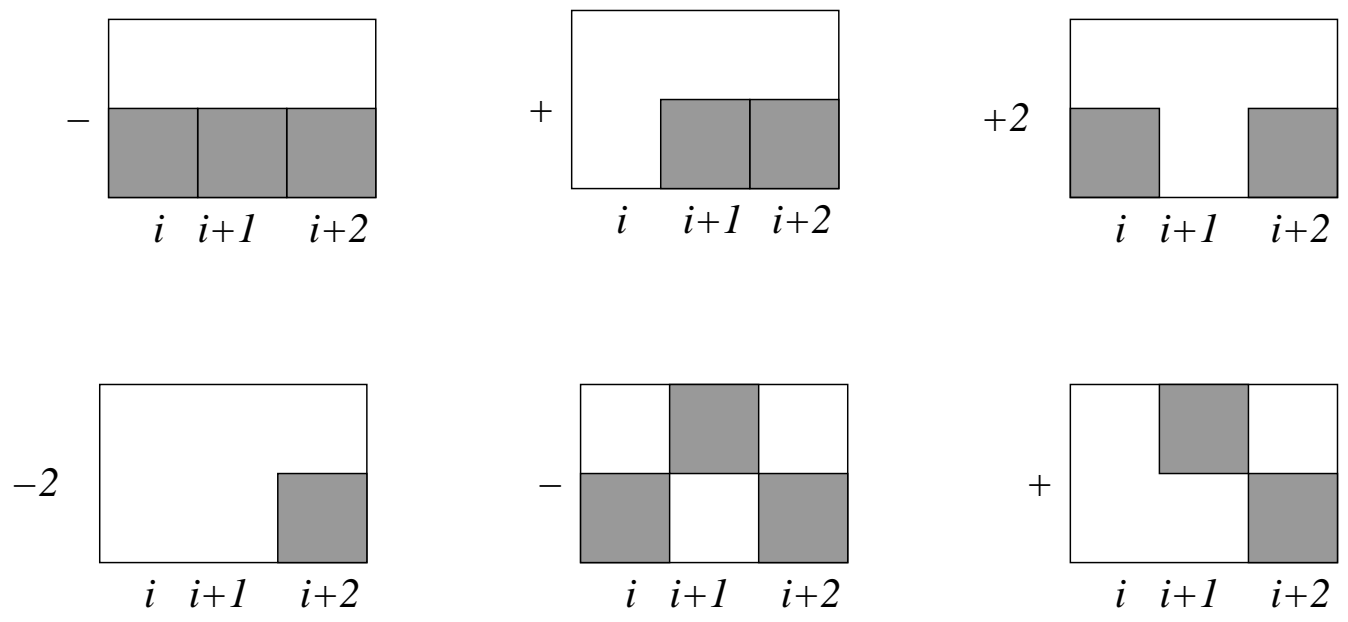

Figure 5: pictorial representation of (9)

According to Section 2.2. the polynomial $O_{k}^{*}$ is the sum of all admissible products of $k$ terms, and each term is either $x_{i}$ or $-X_{j}$. The admissibility condition is that the monomials $x_{i}$ or $X_{j}$ involved are sufficiently distant; what it means precisely is that the respective tiles in Figures 4 and 5, corresponding to these terms, do not overlap. This is a crucial observation.

To summarize, each monomial in $O_{k}^{*}$, after the substitutions (8) and (9), is represented by a collection of $k$ tiles depicted in Figures 4 and 5, taken with the product of their 
respective coefficients. The tiles, that occupy two or three consecutive positions, are placed around a circle having $N$ positions available (if we are concerned with twisted $N$-gons). There may be empty positions left between the tiles.

As a final preparation for the next section, we introduce the following notation: letter $A$ denotes a shaded square in the lower row of a tile ( $p_{i}$ in denominator), letter $B$ a shaded square in the upper row ( $p_{i}$ in numerator), and letter $X$ an empty column in a tile. Thus, the two tiles in Figure 4 correspond to the words $X A$ and $B A$, and the six tiles in Figure 5 to $A A A, X A A, A X A, X X A, A B A$ and $X B A$, respectively. We also use letter $X$ to mean an empty slot between the tiles.

In the next section we reformulate Theorem 3.2 as a statement about a kind of puzzle involving words in letters $\{A, X, B\}$. After we formulate the combinatorial statement, we

prove it. The combinatorial result we prove implies Theorem 3.2, which in turn implies Theorem 1.1

\section{The Puzzle}

\subsection{The Main Result}

Now we are going to extract the main combinatorial information from the discussion at the end of the last section.

We fix some integer $N>0$ and consider the set of length $N$ lists in the letters $\{A, X, B\}$. We consider two lists the same if they are cyclic permutations of each other. We say that a cyclic sentence is an equivalence class of such strings. To illustrate our notation by way of example, $(A X A B A)$ denotes the equivalence class of $A X A B A$. Here $N=5$. We let $\mathcal{S}$ denote the set of such sentences, with the dependence on $N$ understood.

We single out certain strings of letters, and to each of these special words we assign a coefficient and a weight. Here is the list.

$\begin{array}{ccc}\text { word } & \text { coefficient } & \text { weight } \\ X & 1 & 0 \\ X A & 1 & 1 \\ X A A & 1 & 1 \\ X B A & 1 & 1 \\ A X A & 2 & 1 \\ A A A & -1 & 1 \\ B A & -1 & 1 \\ A B A & -1 & 1 \\ X X A & -2 & 1\end{array}$

We say that a parsing of a cyclic sentence is a description of the cyclic sentence as a concatenation of words. For example

$$
(A B A / X A) ; \quad(X A A / B A)
$$


are the only two parsings of $(A B A X A)$. We define the coefficient of a parsing to be the product of the coefficients of the word. We define the weight of the parsing to be the sum of the weights of the words. Both parsings above have coefficient -1 and weight 2 .

For each cyclic sentence $S$, we define $c(S, w)$ to be the total sum of the coefficients of the weight $w$ parsings of $S$. For instance, when $S=(A B A X A)$, we have $c(S, 2)=-2$ and otherwise $c(S, w)=0$. For a more streamlined equation, we define

$$
|S|=\sum_{w=0}^{\infty} c(S, w) t^{w} .
$$

Here $|S|$ is a polynomial in $t$ that encodes all the coefficients at once. For example

$$
|(A B A X A)|=-2 t^{2}
$$

Let $\bar{S}$ denote the cyclic sentence obtained by reversing $S$. In view of Section 3 , Theorem 3.2 is equivalent to the following result.

Theorem 4.1 (Cyclic) We have $|S|=|\bar{S}|$ for all cyclic sentences $S$.

The rest of this chapter is devoted to proving Theorem 4.1 .

\subsection{The Tight Puzzle}

Before we tackle Theorem 4.1, we slightly modify our puzzle for the sake of convenience.

Lemma 4.2 Suppose that $(W)$ contains the string $X B$. Then $|W|=0$.

Proof: No word ends in $B$, and so the string $X B$ must continue as $X B A$. The occurence of $\ldots / X / B A / \ldots$ in any parsing contributes weight 1 and coefficient -1 whereas the occurence of $\ldots / X B A / \ldots$ contributes weight 1 and coefficient 1 . If we have a parsing that involves $\ldots / X B A / \ldots$ we can create a new parsing by replacing the last $\ldots / X B A / \ldots$ with $\ldots / X / B A / \ldots$. These two parsings have the same weight and opposite coefficient, and thereby cancel each other in the total sum.

By Lemma 4.2, we can simply throw out any strings that contain $X B$, and we may drop the word $X B A$ from our list of words. There is a similar cancellation involving $X X A$. Within a parsing, the occurence of $\ldots / X / X A / \ldots$ has weight 1 and coefficient 1 whereas the occurence of $\ldots / X X A / \ldots$ has weight 1 and coefficient -2 . If we have a parsing that involves $\ldots / X X A / \ldots$ we can create a new parsing by replacing the last $\ldots / X X A / \ldots$ with $\ldots / X / X A / \ldots$. The new parsing cancels out "half" of the original. Thus, we may consider an alternate puzzle where the parsing $/ X / X A /$ is forbidden and the word list is 


$\begin{array}{ccc}\text { word } & \text { coefficient } & \text { weight } \\ X & 1 & 0 \\ X A & 1 & 1 \\ X A A & 1 & 1 \\ A X A & 2 & 1 \\ A A A & -1 & 1 \\ B A & -1 & 1 \\ A B A & -1 & 1 \\ X X A & -1 & 1\end{array}$

All we have done is dropped $X B A$ from the list and changed the weight of $X X A$ from -2 to -1 . We call this last puzzle the tight puzzle. Establishing Theorem 4.1 for the tight puzzle is the same as establishing these results for the original one.

\subsection{The Open Version}

As an intermediate step to proving Theorem 4.1, we state a variant of the result. We consider bi-infinite strings in the letters $\{A, B, X\}$, where there are only finitely many $A$ s and $B$ s. We say that two such strings are equivalent if one of them is a shift of the other one. We say that an open sentence is an equivalence class of such strings. We use finite strings to denote sentences, with the understanding that the left and right of the finite string is to be padded with an infinite number of $X \mathrm{~s}$. Thus, $A B A X A$ refers to the bi-infinite sentence ...XX $X B A X A X X \ldots$. We define parsings just as in the cyclic case. For instance, here are all the parsings of this sentence

- $/ A B A / X A /(-1)$

- $/ X X A / B A / X A /(1)$

(Recall that we have forbidden $/ X / X A$.) The first of these have weight 2 and the last one has weight 3 . We have put the coefficients next to the parsings in each case. Our notation is such that the left and right sides of each expression are padded with $\ldots / X / X / \ldots$. Based on the list above, we have

$$
|A B A X A|=-t^{2}+t^{3} .
$$

Here is the variant of Theorem 4.1 for open sentences.

Theorem 4.3 (Open) We have $|S|=|\bar{S}|$ for all open sentences $S$.

Theorem 4.1 implies Theorem 4.3 in a fairly direct way. For instance, suppose we are interested in proving Theorem 4.3 for an open sentence $S$. We say that the span of $S$ is the combinatorial distance between the first and last non- $X$ letter of $S$. For instance, the span of $A B A X A$ is 4 . Supposing that $S$ has span $s$, we simply create a cyclic sentence of length (say) $s+10$ by padding the nontrivial part of $S$ with $X$ s and then taking the cyclic equivalence class. Call this cyclic sentence $S^{\prime}$. We clearly have

$$
|S|=\left|S^{\prime}\right|=\left|\bar{S}^{\prime}\right|=|\bar{S}| \text {. }
$$


The middle equality is Theorem 4.1. The end inequalities are obvious.

Now we turn to the proof of Theorem 4.3. In the next result, $W$ stands for a finite string in the letters $A, B, X$.

Lemma 4.4 (Right Identities) The following identities hold.

1. $|W A A A|+t|W|=0$.

2. $|W X A A|-t|W|=0$.

3. $|W X X A|+t|W|=0$.

4. $|W A X A|-t|W A|-2 t|W|=0$.

5. $|W A B A|+t|W A|+t|W|=0$.

Proof: Consider Identity 1. Any parsing of $W A A A$ must have the form $W / A A A$. But $A A A$ has weight 1 and coefficient -1 . Hence $c(W A A A, w)=-c(W, w-1)$. Also $c(W A A A, 0)=0$. Identity 1 follows immediately from this. Identity 2 and Identity 3 have the same proof.

Consider Identity 4. There are two kinds of parsings of $W A X A$. One kind has the form $W / A X A$ and the other kind has the form $/ W A / X A$. Note that $A X A$ has weight 1 and coefficient 2 and $X A$ has weight 1 and coefficient 1 . From this, we see that $c(W A X A, w)=2 c(W, w-1)+c(W A, w-1)$ for all $w$. Identity 4 follows immediately. Identity 5 has the same proof.

Discussion: If Theorem 4.3 really holds, then the "reverses" of all the identities above should always hold. Let's consider an example in detail. The reverse of Identity 2 above is

$$
|A A X W|-t|W|=0,
$$

for all strings $W$. However, taking $W=A B A$, the weight 3 parsings of $A A X W$ are

- $/ X X A / A X A / B A /(2)$.

- $/ X A A / X A / B A /(-1)$.

As usual, our convention is to leave off the words $\ldots / X / X / \ldots$ on both sides. Adding up the coefficients, we see that $c(A A X W, 3)=1$. At the same time, the only weight 2 parsing of $W$ is

$$
\text { - } / X X A / B A /(1)
$$

Hence $c(W, 2)=1$. This accords with our supposed equality, but the 3 parsings in the one case don't obviously cancel out the 2 parsings in the other. In Lemma 4.4 the various parsings matched up and cancelled each other in an obvious way. However, this does not happen for the reverse identities. Nonetheless, we will prove the reverse identities of Lemma 4.4. 
Lemma 4.5 (Left Identities) The following identities hold.
1. $|A A A W|+t|W|=0$.
2. $|A A X W|-t|W|=0$.
3. $|A X X W|+t|W|=0$.
4. $|A X A W|-t|A W|-2 t|W|=0$.
5. $|A B A W|+t|A W|+t|W|=0$.

Proof: We will prove Identity 5. The other identities have the same proof. First of all, we check computationally that Identity 5 holds (say) for all strings $W$ having length at most 3.

Suppose now that $W$ is a shortest word for which we don't know the result of this lemma. We know that $W$ has length at least 3 , so we can write $W=V R$, where $R$ has length 3 and ends in $A$. Consider the case when $R=A X A$.

By induction, we have

$$
\begin{gathered}
|A B A V|+t|A V|+t|V|=0 . \\
|A B A V A|+t|A V A|+t|V A|=0 .
\end{gathered}
$$

Using Identity 4 of Lemma 4.4 (three times) we have

$$
\begin{gathered}
t|V A X A|=t^{2}|V A|+2 t^{2}|V| . \\
t|A V A X A|=t^{2}|A V A|+2 t^{2}|A V| . \\
|A B A V A X A|=t|A B A V A|+2 t|A B A V| .
\end{gathered}
$$

When we add together the right hand sides of Equations 12, 13, 14, we get 0, thanks to Equations 10] and 11. Hence, when we add the left hand sides of Equations 12, 13, 14, we also get 0 . But this last sum is exactly the identity we wanted to prove.

A similar argument works when $R$ is any of the other 3-letter strings that appear in Lemma 4.4. The only case we haven't considered is the case when $R=X B A$, but these strings are forbidden.

Now that we have Lemma 4.4 and Lemma 4.5, our proof of Theorem 4.3 goes by induction. First of all, we check Theorem 4.3 for all strings having span at most 3 . Suppose then that $W$ is the shortest open sentence for which we do not know Theorem 4.3. We can write $W=V R$ where $R$ is some string of length 3 that ends in $A$.

Let's consider the case when $R=X A A$. Then we have

$$
|W|=|V X A A|=t|V|=t|\bar{V}|=|A A X \bar{V}|=|\bar{W}| .
$$

The second equality is Identity 2 of Lemma 4.4. The third equality is the induction assumption. The fourth equality is Identity 2 of Lemma 4.5. A similar argument works when $R$ is any of the 3 letter strings in Lemma 4.4. The final case, $R=X B A$, is forbidden.

This completes the proof of Theorem 4.3. 


\subsection{The Cyclic Case}

We need to mention another convention before we launch into the cyclic case. Besides cyclic and open sentences, there is one more case we can consider. We introduce the notation $[W]$ to denote an open word whose parsings cannot be created by padding $X \mathrm{~s}$ onto the left and right of $W$. We will illustrate what we have in mind by way of example. Setting $W=A B A X A$, the parsings of the open string $W$ are

$$
/ A X A / B A / ; \quad / X X A / X / A B A \text {. }
$$

However, the second parsing involves two $X \mathrm{~s}$ that have been padded onto the left of $W$. Only the first parsing of $W$ is also a parsing of the locked string $[W]$. We let $|[W]|$ be the polynomial that encodes the weights and coefficients of all the parsings of $[W]$.

Lemma 4.6 Theorem 4.1 holds for any cyclic word $W$ with no $X$ in it.

Proof: To avoid some messy notation, we will consider an example. The example is sufficiently complex that it should illustrate the general proof. Suppose that

$$
W=\left(B A^{2} B A^{5} B A^{1} B A^{7}\right) .
$$

Here, for instance $A^{2}=A A$. Any parsing of $W$ must have the breaks

$$
W=\left(B A / A^{1} B A / A^{4} B A / A^{0} B A / A^{6}\right) .
$$

The point is that we must have a break after each $B A$. From this, we see that

$$
|W|=\left|\left[A^{6} B A\right]\right| \times|[A B A]| \times\left|\left[A^{4} B A\right]\right| \times\left|\left[A^{0} B A\right]\right| .
$$

To get the list of exponents on the right hand side of this product, we simply decrement each exponent in Equation 15 by one. But we would get the same list of exponents (perhaps in a different order) when considering the reverse word $\bar{W}$.

Below we prove the following result.

Lemma 4.7 The relation

$$
|(W X)|+2|(W B)|-|W|=0
$$

holds for all open words $W$.

Lemma 4.7 allows us to finish the proof of Theorem 4.1. Our proof goes by induction on the number of $X s$ in the word $W$. Let $W$ be a word having the smallest number of $X \mathrm{~s}$, for which we do not know Theorem 4.1

After cyclically permuting the letters in $W$, we can write $W=V X$. By Lemma 4.7 we have

$$
|(W)|=|(V X)|=-2|(V B)|+|V| .
$$


Applying Lemma 4.7 to $\bar{W}$, we have

$$
|(\bar{W})=|(\bar{V} X)|=-2|(\bar{V} B)|+| \bar{V}|=-2| \overline{V B}|+| \bar{V} \mid .
$$

Setting $Y=V B$, we have

$$
|(W)|=-2|(Y)|+|V|=-2|(\bar{Y})|+|\bar{V}|=|(\bar{W})| .
$$

The middle equality comes from Theorem 4.3 (to handle $V$ ) and the induction assumption (to handle $Y$.)

\subsection{Some Auxilliary Relations}

It remains only to prove Lemma 4.7. We will establish some auxilliary relations in this section, and then use them in the next section to prove Lemma 4.7 .

Lemma 4.8 For any string $W$,

$$
|X X W|=|W|
$$

Proof: This is a tautology.

Lemma 4.9 For any string $W$,

$$
|W|=|[X W]|-|[A X W]| .
$$

Proof: The proof makes use of the right identities from Lemma 4.4 and is similar to the proof of Theorem 4.3. One first checks the statement for all strings of span three, and then argues inductively on the span. The induction step is proved using the right identities from Lemma 4.4 that hold verbatim for locked strings as well.

To illustrate the idea, we assume that $W$ ends with $A X A$, that is, $W=V A X A$. Then, by Identity 4 of Lemma 4.4.

$$
\begin{gathered}
|W|=|V A X A|=t|V A|+2 t|V|, \\
\mid[X W|=|[X V A X A]|=t|[X V A]|+2 t|[X V] \mid,
\end{gathered}
$$

and

$$
|[A X W]|=|[A X V A X A]|=t|A X V A|+2 t|[A X V]| .
$$

By the induction assumption,

$$
|V A|=|[X V A]|-|[A X V A]|, \quad|V|=|[X V]|-|[A X V]|,
$$

and the result follows for $W$. 
Lemma 4.10 For any string $W$,

$$
|(A X A W)|=2 t|[W]|+|[X A W A]| .
$$

Proof: Either a parsing of the cyclic word $(A X A W)$ contains the string $A X A$, or there is a break after the first $A$ in $(A X A W)$. The former case corresponds to the first term, $2 t|[W]|$, and the latter case to the second, $|[X A W A]|$.

Lemma 4.11 For any string $W$,

$$
|(A B A W)|=|[W A B A]| .
$$

Proof: There must be a break after second $A$ in $(A B A W)$, and this provides a one-to-one correspondence between the parsings of $(A B A W)$ and $[W A B A]$.

\subsection{Proof of Lemma 4.7}

Lemma 4.12 Lemma 4.7 holds if $W$ does not start and end with $A$.

Proof: This is a case-by-case analysis. Suppose that $W=V X$ for some word $V$. Then $(W X)=(V X X)$. Since we must have a break between $V$ and $X X$, it follows that

$$
|(V X X)|=|X X V|=|V|=|W| .
$$

(the second equality holds by Lemma 4.8). On the other hand,

$$
|(W B)|=|(V X B)|=0
$$

since the combination $X / B A$ is prohibited. The claim of Lemma 4.7 follows. Similarly, if $W=X V$ for some word $V$ then $(W X)=(V X X)$, and the same argument applies. If $W=V B$ then then each term in the equality of Lemma 4.7 vanishes. The same holds if $W=B V$. Finally, if $W$ ends in $B$, all terms in Lemma 4.7 are trivial.

The only remaining case is when $W=A V A$ for some word $V$. What we need to prove is

$$
|(A X A V)|+2|(A B A V)|-|A V A|=0 .
$$

By Lemma 4.10,

$$
|(A X A V)|=2 t|[V]|+|[X A V A]|
$$

By Lemma 4.11,

$$
|(A B A V)|=|[V A B A]|
$$


By Lemma 4.9.

$$
|A V A|=|[X A V A]|-|[A X A V A]| .
$$

Therefore, the left hand side of (16) equals

$$
2 t|[V]|+|[X A V A]|+2|[V A B A]|-|[X A V A]|+|[A X A V A]| .
$$

By Identity 5 of Lemma 4.4 for locked words,

$$
|[V A B A]|=-t|[V A]|-t|[V]| .
$$

Finally,

$$
|[A X A V A]|=2 t|[V A]|
$$

since a parsing of $[A X A V A]$ must start with $A X A$. It follows that (17) equals

$$
2 t|[V]|+|[X A V A]|-2 t|[V A]|-2 t|[V]|-|[X A V A]|+2 t|[V A]|=0,
$$

as needed.

This completes the proof of Lemma 4.7.

\section{References}

[B] G. M. Beffa, On Generalizations of the Pentagram Map: Discretizal of AGD Flows, preprint 2011

[G] M. Glick, The Pentagram map and Y-Patterns, Adv. in Math. 227 (2011), 10191045.

[M] Th. Motskin, The Pentagon in the projective plane, and a comment on Napier's rule, Bull. Amer. Math. Soc. 52 (1945), 985-989.

[OST1] V. Ovsienko, R. E. Schwartz, S. Tabachnikov, The Pentagram Map: A Discrete Integrable System, Comm. Math. Phys. 299 (2010), 409-446.

[OST2] V. Ovsienko, R. E. Schwartz, S. Tabachnikov, Quasiperiodic motion for the pentagram map, Electr. Res. Announ. Math. 16 (2009), 1-8.

[S1] R. Schwartz, The Pentagram Map J. Experiment. Math. 1 (1992), 71-81.

[S2] R. Schwartz, Recurrence of the Pentagram Map, J. Experiment. Math. 110 (2001), 519-528.

[S3] R. Schwartz, Discrete Monodromy, Pentagrams, and the Method of Condensation, J. Fixed Point Theory Appl. 3 (2008), 379-409.

[Sol] F. Soloviev, Integrability of the Pentagram Map, arXiv:1106.3950.

[ST] R. Schwartz, S. Tabachnikov, Elementary Surprises in Projective Geometry, Math. Intelligencer 32 (2010) No 3, 31-34. 